\title{
Characterization of coagulase-negative staphylococcal isolates from blood with reduced susceptibility to glycopeptides and
} therapeutic options

\author{
Silvia Natoli*†1,5, Carla Fontana ${ }^{\dagger 2}$, Marco Favaro $^{2}$, Alberto Bergamini $^{3}$, \\ Gian Piero Testore ${ }^{3}$, Silvia Minelli², Maria Cristina Bossa ${ }^{2}$, Mauro Casapulla1, \\ Giorgia Broglio ${ }^{1}$, Angela Beltrame ${ }^{3}$, Laura Cudillo ${ }^{4}$, Raffaella Cerretti ${ }^{4}$ and \\ Francesca Leonardis ${ }^{\dagger}$
}

\begin{abstract}
Address: ${ }^{1}$ Intensive Care Unit, Department of Surgery, "Tor Vergata" University of Rome, Via Montpellier 1, 00133 Rome, Italy, ${ }^{2}$ Clinical Microbiology Laboratories, Polyclinic of Tor Vergata, Viale Oxford 81, 00133 Rome, Italy, ${ }^{3}$ Infectious Diseases, Department of Public Health, "Tor Vergata" University of Rome, Via Montpellier 1, 00133 Rome, Italy, ${ }^{4}$ Haematology, Department of Biopathology, Polyclinic of Tor Vergata, Viale Oxford 81, 00133 Rome, Italy and 5ipartimento delle Emergenze e di Accettazione, di Medicina Critica del Dolore e delle Scienze Anestesiologiche -UOSD Terapia Intensiva, Policlinico Tor Vergata, Vale Oxford 81, 00133 Roma, Italy

Email: Silvia Natoli* - silvia.natoli@ptvonline.it; Carla Fontana - carla.fontana@uniroma2.it; Marco Favaro - favaro@uniroma2.it; Alberto Bergamini - bergamini@med.uniroma2.it; Gian Piero Testore - testore@med.uniroma2.it; Silvia Minelli - silvia.minelli@ptvonline.it; Maria Cristina Bossa - cristi14@hotmail.com; Mauro Casapulla - mauro.casapulla@libero.it; Giorgia Broglio - giorgia.broglio@ hotmail.it; Angela Beltrame - beltrame.angela@gmail.com; Laura Cudillo - cudillo@med.uniroma2.it; Raffaella Cerretti - raffaella.cerretti@ptvonline.it; Francesca Leonardis - leonardis@med.uniroma2.it

* Corresponding author †Equal contributors
\end{abstract}

Published: 4 June 2009

BMC Infectious Diseases 2009, 9:83 doi:10.1 186/1471-2334-9-83

This article is available from: http://www.biomedcentral.com/I47I-2334/9/83

(C) 2009 Natoli et al; licensee BioMed Central Ltd.

This is an Open Access article distributed under the terms of the Creative Commons Attribution License (http://creativecommons.org/licenses/by/2.0), which permits unrestricted use, distribution, and reproduction in any medium, provided the original work is properly cited.
Received: I December 2008 Accepted: 4 June 2009

which permits unrestricted use, distribution, and reproduction in any medium, provided the original work is properly cited.

\begin{abstract}
Background: Coagulase-negative staphylococci (CoNS) are a major cause of nosocomial blood stream infection, especially in critically ill and haematology patients. CoNS are usually multidrugresistant and glycopeptide antibiotics have been to date considered the drugs of choice for treatment. The aim of this study was to characterize CoNS with reduced susceptibility to glycopeptides causing blood stream infection (BSI) in critically ill and haematology patients at the University Hospital Tor Vergata, Rome, Italy, in 2007.

Methods: Hospital microbiology records for transplant haematology and ICU were reviewed to identify CoNS with elevated MICs for glycopeptides, and isolates were matched to clinical records to determine whether the isolates caused a BSI. The isolates were tested for susceptibility to new drugs daptomicin and tigecycline and the genetic relationship was assessed using f-AFLP.

Results: Of a total of 17,4I 8 blood cultures, I,609 were positive for CoNS and of these, 87 (5.4\%) displayed reduced susceptibility to glycopeptides. Clinical review revealed that in 13 cases (7 in haematology and 6 in ICU), CoNS with reduced susceptibility to glycopeptides were responsible for a BSI. Staphylococcus epidermidis was the causative organism in II instances and Staphylococcus haemolyticus in 2 . The incidence of oxacillin resistance was high (77\%), although all isolates remained susceptible to linezolid, daptomycin and tigecycline. Fingerprinting of CoNS identified one clonal relationship between two isolates.
\end{abstract}


Conclusion: Multi-resistant CoNS with reduced susceptibility to glycopeptides, although still relatively infrequent in our hospital, are emerging pathogens of clinical concern. Surveillance by antibiotyping with attention to multi-resistant profile, and warning to clinicians, is necessary.

\section{Background}

Coagulase-negative staphylococci (CoNS) are normal commensals of the skin, anterior nares, and ear canals of humans. They have long been considered as nonpathogenetic, and were rarely reported to cause severe infections. However, as a result of the combination of increased use of intravascular devices and an increase in the number of hospitalized immunocompromised patients, CoNS have emerged as a major cause of nosocomial bloodstream infections [1-3].

CoNS, primarily Staphylococcus haemolyticus and Staphylococcus epidermidis, are often resistant to multiple antibiotics, and glycopeptides have been considered the drugs of choice for the management of infections caused by these organisms [4,5]. However, widespread use of glycopeptides recently has led to the emergence of CoNS isolates with decreased susceptibility to glycopeptides, displaying a minimal inhibitory concentration (MIC) of 4-8 $\mathrm{mg} / \mathrm{L}$ for vancomycin and/or a MIC of 8-16 for teicoplanin [68]. Although acquired resistance to glycopeptides was first documented in CoNS in 1986 [9], attention was focussed on glycopeptide resistance in enterococci $[10,11]$, due in large part to the historically relatively infrequent occurrence of CoNS infections.

In the late 1990s, the emergence of glycopeptides resistant Staphylococcus aureus in Japan [12] and in the USA [13] and later reports on hetero-resistance to glycopeptides in MRSA $[14,15]$ raised the issue of the limited therapeutic options remaining for these Gram positive multi-resistant bacteria that largely cause nosocomial infections. The extensive clinical isolation of multi-resistant CoNS, especially from blood, poses similar clinical concerns and challenges.

At the University Hospital of Tor Vergata, Rome, Italy, CoNS are most frequently isolated from blood cultures taken from haematologic patients and from critically ill patients admitted to the intensive care unit (ICU). Occasionally, these CoNS isolates display a reduced susceptibility to glycopeptides. Since the majority of these isolates can be considered as contaminant, the incidence of CoNS with reduced sensitivity to glycopeptides actually causing bloodstream infections (BSI) is difficult to determine. The objective of this study was to investigate whether glycopeptide-resistant CoNS represent a significant clinical concern by examining CoNS isolates and patient records for 2007. The second aim was to determine whether the infecting bacteria were clonally related and to review the therapeutic options.

\section{Methods}

This study was approved by local Ethical Committee (University of Rome - Polyclinic Tor Vergata) on march 2008. We reviewed University Hospital Tor Vergata laboratory records in 2007 to identify any CoNS isolated from blood having a minimal inhibitory concentration (MIC) of $\geq 2$ $\mu \mathrm{g} / \mathrm{mL}$ for vancomycin and/or an MIC of $\geq 8 \mu \mathrm{g} / \mathrm{mL}$ for teicoplanin. The search was limited to the transplant haematology ward and the ICU where patient and clinical data are routinely collected in a local database in which every infective event is catalogued. All clinical isolates were registered and stored at $-80^{\circ} \mathrm{C}$ in defibrinated bovine blood. Only non-duplicate blood stream isolates included in the analysis.

We obtained information on the clinical course and outcome, microbiological outcome, any antecedent use of antimicrobial agents and actual therapy for each patient who had a blood stream infection with a CoNS with reduced susceptibility to glycopeptides.

Criteria used to categorize isolates as contaminants versus blood stream infection were the following:

1) fever or signs of sepsis at the time of blood culture;

2) isolation of the same potential skin contaminant from two or more blood cultures drawn on separate occasions within a 48-hour period AND isolated from a patient with an intravascular access device inserted at least 48 hours before AND physician institutes appropriate antimicrobial therapy;

3) absence of any other possible site of infection;

To define whether an infection was catheter-related, one of the following criteria had to be satisfied:

1) catheter tip was positive for the same agent retrieved at a peripheral vein

2) blood culture drawn from catheter hubs revealed the same agent isolated from peripheral vein but at least 120 minutes before the latter. 
All the infectious events were classified by an expert infectivologist in the local database as the microbiology results were obtained.

\section{Laboratory methods}

Biochemical identifications and antibiotic susceptibility tests were performed using the VITEK 2 automated system (bioMerieux) following the manufacturer's instructions. Susceptibility to tygeciclin and daptomycin was tested using the appropriate E-test ( $\mathrm{AB}$ Biodisk) following the manufacturer's instructions. The strains were first induced to express glycopeptides resistance by culture on Vancoagar screen plate, according to the Clinical and Laboratory Standards Institute (appendix B) and CDC guidelines, containing $6 \mu \mathrm{g} / \mathrm{mL}$ of vancomycin [16,17]. Then they were tested with authomated system as well as by E-test. The MIC $(\mu \mathrm{g} / \mathrm{mL})$ interpretative standard for vancomycin and teicoplanin were those suggested by EUCAST (respectively: $\leq 4$ susceptible, $\geq 8$ resistant).

\section{Amplified Fragment Length Polymorphism}

The genetic relationship among the isolates was determined by fingerprinting using the commercial kit f-AFLP Microbial fingerprinting (Applera; Foster City, California) according to the manufacturer's instructions using as primers: AFLP primer MseI-CC, MseI-CG and AFLP FAM primer EcoRI-O. The f-AFLP reactions were loaded and run on the ABI 310 DNA genetic analyzer (Applera). Each f-AFLP reaction was analysed using Genescan software and Genographer program version 1.6.0 (kindly provided by James J. Benham, http://hordeum.oscs.montana.edu/ genographer; Copyright C 1999 Montana State University). Cluster analysis was performed using the unweighted pair group method with the averages algorithm (UPGMA) [18]. The percentage similarity between patterns was calculated using the Dice correlation coefficient [19].

\section{Results}

\section{Patients and isolates}

A total of 17,418 blood samples were screened in 2007 , 14,195 from the transplant haematology ward and 3223 from ICU patients (Table 1). For the transplant haematology samples, 1959 were blood culture-positive and of these, 1383 (70.6\%) yielded CoNS. Similarly, CoNS were present in $47.3 \%(226 / 478)$ of culture-positive blood samples from the ICU. Overall, using criteria defined in the methods section, 92 out of 1609 CoNS positive blood cultures $(5.7 \%)$ were considered to be associated with infection. Particularly in the transplant haematology ward 62 out of 1386 (4\%) CoNS positive blood cultures caused a BSI while in ICU, the BSI were 30 out of 226 CoNS isolates $(13 \%)$. In total, 87 CoNS isolates (69 S. epidermidis, $18 \mathrm{~S}$. haemolyticus) displayed a reduced susceptibility to glycopeptides, a frequency of 5.4\%. Of these, 7 and 6 were considered to be associated with infection in patients from the transplant haematology ward and ICU, respectively. Infections caused by CoNS with reduced susceptibility to glycopeptides represent $11 \%$ and $20 \%$ of all CoNS related BSI in haematology ward and ICU respectively.

Clinical data for patients infected by CoNS with reduced susceptibility to glycopeptides are presented in Tables 2 and 3. For transplant haematology patients, all had a central vein device and were bone marrow transplant recipients (Table 2). Not all patients met the full criteria for catheter related blood stream infection (CR-BSI). Nevertheless the infective event was classified as a blood stream infection in the database. For all patients a combination antimicrobial therapy was established according to the therapy protocols of the ward. In all cases microbiology eradication was reached. For ICU patients, all were being treated for one other infection at the time of blood-culture, and all had a central vein device. In patient 9 it was not possible to define whether the BSI was catheter related because the patient died before completing the diagnosis. In ICU the patients were treated with linezolid according to susceptibility test. In all cases, except for patient 9 who was not treated, microbiological eradication was obtained. Overall, only 2 patients (patients 2 and 3, both from the transplant haematology ward) had recorded previous exposure to glycopeptides.

The majority of isolates $(11 / 13)$ were identified as $S$. epidermidis, with the remaining two being $S$. haemolyticus, both of which were isolated from transplant haematology ward patients (Table 4). All isolates demonstrated reduced sensitivity to vancomycin ( $\mathrm{MIC} \geq 2 \mu \mathrm{g} / \mathrm{mL}$ ) whereas 7 isolates demonstrated reduced sensitivity to teicoplanin

Table I: Results of blood culture screening.

\begin{tabular}{|c|c|c|c|c|c|}
\hline & \multirow[t]{2}{*}{$\begin{array}{l}\text { Blood cultures screened } \\
\text { (no. positive) }\end{array}$} & \multicolumn{2}{|c|}{ Blood cultures yielding CoNS } & \multicolumn{2}{|c|}{$\begin{array}{c}\text { Strains with reduced susceptibility to } \\
\text { glycopeptides }\end{array}$} \\
\hline & & Total & Associated with infection & Total & Associated with infection \\
\hline $\begin{array}{l}\text { Transplant haematology } \\
\text { ward }\end{array}$ & 14,195 (1959) & 1383 & 62 & 76 & 7 \\
\hline $\mathrm{ICU}$ & $3223(478)$ & 226 & 30 & 11 & 6 \\
\hline
\end{tabular}


Table 2: Characteristics of patients from transplant haematology ward infected with CoNS displaying reduced susceptibility to glycopeptides

\begin{tabular}{|c|c|c|c|c|c|c|c|c|c|c|}
\hline Pat. & Age & $\begin{array}{c}\text { Underlying } \\
\text { disease }\end{array}$ & $\begin{array}{l}\text { Antimicrobial } \\
\text { prophylaxis }\end{array}$ & $\begin{array}{l}\text { Neutrophyl } \\
\text { count } / \mathrm{mm}^{3}\end{array}$ & $\begin{array}{c}\text { Days from } \\
\text { transplantation }\end{array}$ & $\begin{array}{l}\text { Immuno- } \\
\text { suppression }\end{array}$ & Therapy & $\begin{array}{l}\text { Catheter } \\
\text { removal }\end{array}$ & CR-BSI & $\begin{array}{c}\text { Micro- } \\
\text { biological } \\
\text { eradication }\end{array}$ \\
\hline 1 & 29 & ALL & no & 2300 & 43 & ciclosporin & $\begin{array}{c}\text { linezolid-pip/ } \\
\text { tazo-amika }\end{array}$ & no & $?$ & yes \\
\hline 2 & 40 & MM & no & 1380 & 128 & ciclosporin & $\begin{array}{l}\text { teicoplanin-pip/ } \\
\text { tazo-amika }\end{array}$ & no & $?$ & yes \\
\hline 3 & 34 & AML & levofloxacin & 10 & 9 & no & $\begin{array}{l}\text { linezolid-pip/ } \\
\text { tazo-amika }\end{array}$ & yes & yes & yes \\
\hline 4 & 20 & $H D$ & no & 6200 & 3 & no & $\begin{array}{l}\text { teicoplanin-pip/ } \\
\text { tazo-amika }\end{array}$ & no & ? & yes \\
\hline 5 & 52 & $\mathrm{NHL}$ & levofloxacin & 10 & 5 & no & $\begin{array}{l}\text { teicoplanin-pip/ } \\
\text { tazo-amika }\end{array}$ & yes & yes & yes \\
\hline 6 & 54 & ALL & no & 1000 & 3 & ciclosporin & teicoplanin & yes & yes & yes \\
\hline 7 & 58 & MM & no & 3700 & 30 & no & $\begin{array}{l}\text { teicoplanin-pip/ } \\
\text { tazo-amika }\end{array}$ & yes & yes & yes \\
\hline
\end{tabular}

$\mathrm{ALL}=$ acute lymphocitic leukaemia; $\mathrm{AML}=$ acute myeloid leukaemia; $\mathrm{MM}=$ multiple myeloma; $\mathrm{HD}=$ Hodgkin's disease; $\mathrm{NHL}=$ non-Hodgkin's lymphoma; $\mathrm{MUD}=$ matched unrelated donor; pip/tazo = piperacillin/tazobactam; amika = amikacin; CR-BSI = catheter related blood stream infection; ? = not confirmed

(MIC $\geq 8 \mu \mathrm{g} / \mathrm{mL})$. The MLSB phenotype, and resistance to oxacillin ( $\mathrm{MIC} \geq 4 \mu \mathrm{g} / \mathrm{mL}$ ) were widespread, each being found in $10(77 \%)$ isolates. All strains remained sensitive to daptomycin, tigecycline and linezolid (Table 4). All strains were also able to grow on Vanco-screen agar according to CLSI algorithm. This means that subpopulations in each strain grow in the presence of $\geq 6 \mathrm{mg} / \mathrm{L}$ of vancomycin. Accordingly these strains display heteroresistance and may be associated with therapeutic failure.

The ratio of $S$. epidermidis to $S$. haemolyticus in the strains associated with infection was $11 / 2=5.5$. Overall, including resistant CoNS considered to be contaminants, the ratio $S$. epidermidis to $S$. haemolyticus was $69 / 18=3.8$

There was no obvious trend in isolation of CoNS with reduced sensitivity to glycopeptides across the year of study (Fig. 1).

\section{Genomic analysis}

As shown by f-AFLP profile analysis (Fig. 2), the genetic relatedness among the 13 isolates was low (overall similarity $<65 \%$ ). Cluster analysis revealed two clusters corresponding to transplant haematology ward isolates (overall similarity approximately $70 \%$ ) and ICU isolates (overall similarity 73\%). In the ICU-cluster, two isolates (from patients 11 and 12) had a similarity of 95\% and were clonally related. The isolate from patient 11 was retrieved in the month of august while the CoNS from patient 12 was isolated in the month of November. The two patients were admitted and confined in bed in different periods.

\section{Discussion}

All the patients were at increased risk for the development of CoNS infections for different reasons. Patients from the transplant haematology ward were neutropenic or were

Table 3: Characteristics of patients from ICU infected with CoNS displaying reduced susceptibility to glycopeptides.

\begin{tabular}{|c|c|c|c|c|c|c|c|}
\hline Patient & Age & $\begin{array}{l}\text { Diagnosis on } \\
\text { admission }\end{array}$ & $\begin{array}{l}\text { Therapy on day of } \\
\text { blood cultures }\end{array}$ & CR-BSI & Catheter removal & Treatment & $\begin{array}{c}\text { Micro-biological } \\
\text { eradication }\end{array}$ \\
\hline 8 & 59 & $\begin{array}{l}\text { Pseudomonas aeruginosa } \\
\text { pneumonia }\end{array}$ & $\begin{array}{c}\text { piperacillin/tazobactam }+ \\
\text { claritromicin }+ \\
\text { teicoplanin }\end{array}$ & yes & yes & linezolid & yes \\
\hline 9 & 83 & Abscess of psoas & $\begin{array}{c}\text { piperacillin/tazobactam }+ \\
\text { metronidazole }+ \\
\text { teicoplanin }\end{array}$ & $?$ & no & none & $?$ \\
\hline 10 & 53 & $\begin{array}{l}\text { Post neurosurgery } \\
\text { cerebral abscess }\end{array}$ & meropenem + teicoplanin & yes & yes & linezolid & yes \\
\hline II & 59 & $\begin{array}{l}\text { MDR } P \text {. aeruginosa } \\
\text { pneumonia }\end{array}$ & meropenem & $?$ & no & linezolid & yes \\
\hline 12 & 85 & $\begin{array}{c}\text { Acinetobacter baumannii } \\
\text { pneumonia }\end{array}$ & colimicin & yes & yes & linezolid & yes \\
\hline 13 & 66 & $\begin{array}{c}\text { Sepsis from infected } \\
\text { flebitis in multiple trauma } \\
\text { patient }\end{array}$ & fluconazole & yes & yes & linezolid & yes \\
\hline
\end{tabular}

CR BSI = catheter related blood stream infection; ? = not confirmed 
Table 4: Characteristics of CoNS clinical isolates associated with infection.

\begin{tabular}{|c|c|c|c|c|c|c|c|c|}
\hline Pat & Species & MIC VAN & MIC TEIC & MLSB phenoptype & MIC OXA & MIC DAP & MIC TIG & MIC LZD \\
\hline \multicolumn{9}{|c|}{ Transplant haematology ward isolates } \\
\hline 1 & S. epidermidis & 2 & 2 & yes & 4 & 0.023 & 0.047 & 2 \\
\hline 2 & S. epidermidis & 2 & 4 & yes & 0.5 & 0.047 & 0.047 & 1 \\
\hline 3 & S. epidermidis & 2 & 4 & yes & 4 & 0.094 & 0.064 & 1 \\
\hline 4 & S. haemolyticus & 2 & 8 & yes & 4 & 0.125 & 0.047 & 2 \\
\hline 5 & S. epidermidis & 2 & 4 & no & 0.25 & 0.047 & 0.032 & 1 \\
\hline 6 & S. haemolyticus & 2 & 8 & yes & 4 & 0.064 & 0.047 & 2 \\
\hline 7 & S. epidermidis & 4 & 16 & no & 0.25 & 0.125 & 0.047 & 2 \\
\hline \multicolumn{9}{|c|}{ ICU isolates } \\
\hline 8 & S. epidermidis & 2 & 4 & yes & 4 & 0.023 & 0.047 & I \\
\hline 9 & S. epidermidis & 2 & 16 & yes & 4 & 0.047 & 0.047 & 2 \\
\hline 10 & S. epidermidis & 4 & $\geq 32$ & no & 4 & 0.094 & 0.064 & 1 \\
\hline 11 & S. epidermidis & 2 & 16 & yes & 4 & 0.125 & 0.047 & 2 \\
\hline 12 & S. epidermidis & 2 & 16 & yes & 4 & 0.064 & 0.047 & 1 \\
\hline 13 & S. epidermidis & 2 & 4 & yes & 4 & 0.047 & 0.032 & 2 \\
\hline
\end{tabular}

MIC values are presented as $\mu \mathrm{g} / \mathrm{mL}$. VAN = vancomycin; $T E I C=$ teicoplanin; $M L S B=$ resistance to macrolides, lincosamides and type $B$ streptogramins; OXA = oxacillin; DAP = daptomycin; TIG = tigecycline; $L Z D=$ linezolid.

\section{Coagulase-negative staphylococci per month}



Figure I

Isolation frequency by month of CoNS strains with reduced susceptibility to glycopeptides in 2007. Data are presented for all isolates; the 13 isolates deemed to be associated with infection are indicated by asterisks. Blue asterisks indicate clonal relationship. 


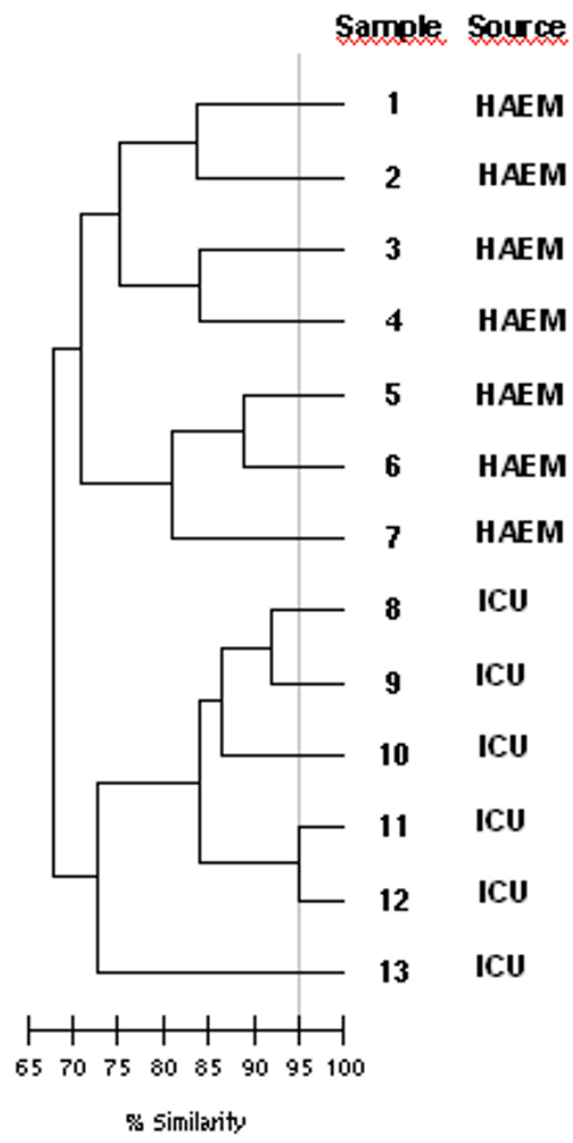

Figure 2

f-AFLP analysis of the genetic relatedness of CoNS isolates with reduced susceptibility to glycopeptides.

receiving immunosuppressant drugs, while patients from the ICU were critically ill and were being treated with broad spectrum antibiotics for different infections. Importantly, they all had a central vein catheter. One observation of concern is that only two of the thirteen patients had previous exposure to glycopeptides. This is in contrast to other reports describing the selection of resistance after exposure to vancomycin or teicoplanin in CoNS $[8,20]$. In the current study, the great number of CoNS isolated from blood were considered to be contaminants, particularly in the haematology ward where blood cultures are drawn for routine for surveillance other than to diagnose infection. In this ward therapy protocols schedule an association of antimicrobials on signs of infections. This may lead to underestimate the frequency of CR-BSI interfering with cultures. In ICU blood cultures are drawn on signs of infection, so the number of clinically relevant CoNS out of the total amount of blood cultures is higher, although the level of contamination is also high. It can be very difficult to draw blood from venipuncture in haematology or critically ill patients, so a certain level of contamination is probably unavoidable, but our data impose to be more strict in sending in blood for cultures in order to limit false positive cultures that may lead to overtreatment.

Our data support the idea that CoNS with reduced susceptibility to glycopeptides are endemic in these wards and are maybe resident on skin and nares of personnel and patients. Indeed, genomic analysis revealed that different strains were responsible for most infections. Only two isolates displayed a clonal relationship. As a direct contact between the two patients was excluded, ward or personnel contamination should be implicated. Nevertheless, as far as we are aware, there is no indication for screening and decontamination of CoNS in patients and personnel from critical areas such as the haematology ward and the ICU. This could be an issue for further studies.

After this study, the actual therapy strategy in the two wards is to start empirical treatment with glycopeptides if CR-BSI is suspected and promptly shift to a different drug according to susceptibility test if the diagnosis is confirmed or if treatment fails. Nevertheless if the signs of infection are severe or if clinical conditions do not allow to wait, therapy with linezolid is started and eventually de-escalated on sensitivity report.

The majority of isolates were $S$. epidermidis and this species was slightly more frequently associated with BSI than $S$. haemolyticus. It was widely accepted that $S$. haemoyiticus is uniquely predisposed among CoNS to develop glycopeptides resistance as this was the first CoNS species in which vancomycin and teicoplanin resistance was identified [21]. Nowadays $S$. epidermidis is often reported to be a nosocomial pathogen bearing multi-resistance [22-24]. Moreover there are many studies suggesting the possible relationship between methicillin resistance and reduced susceptibility to glycopeptides in CoNS $[9,25,26]$.

Not all clinical isolates demonstrated MICs for vancomycin and teicoplanin in the intermediate susceptibility range, but they were all shifted towards the cut-off level. Moreover, these isolates were also able to grow on Vancoscreen agar. It has been previously shown that staphylococci able to grow on Vanco-screen agar may display heteroresistance to glycopeptides [27]. Heteroresistance was first defined as the presence of $>10^{-6}$ stable cell subpopulations of a strain that is apparently susceptible to vancomycin on the basis of conventional criteria, but for which the vancomycin MIC for the subpopulation of cells is greater than or equal to $8 \mathrm{mg} /$ liter [12]. Since 2006 CLSI breakpoints have changed, actual definition might be that although the MIC for the entire population is within the range of susceptibility according to the CLSI criteria, subpopula- 
tions grow in the presence of $\geq 4 \mathrm{mg} / \mathrm{L}$ of vancomycin. The hetero-resistant phenotype may be associated with treatment failure and/or may be precursor of glycopeptide resistance and should be considered in both empirical and rational therapy decisions.

In our series, CoNS remained homogeneously susceptible in vitro to linezolid, tigecycline and daptomycin. Of these three drugs only daptomycin is suitable for the treatment of BSI, given its pharmacokinetic profile. Linezolid was used off label to treat most patients. The use of linezolid for the treatment of catheter related Gram positive blood stream infections is still a matter of discussion, as there have been suggestions that it is associated with a higher mortality rate depending on the type of infection [28]. Nevertheless, in a recent cohort study, empiric therapy with linezolid was associated with greater survival and more successful microbiological eradication compared to standard therapy in the treatment of methicillin-resistant $S$. aureus bacteremia [29]. Further, in a pharmacoeconomic evaluation study linezolid was considered more efficient than teicoplanin in the treatment of Gram-positive bacteremia [30].

Tigecycline is not registered for the treatment of bacteremia, and its use should be limited to real clinical necessity to preserve its activity against multi-resistant Gram negative bacteria. Clinical efficacy of daptomycin has been demonstrated in patients with $S$. aureus bacteremia [31]. To our knowledge no clinical data exist on daptomycin efficacy against CoNS with elevated MICs for glycopeptides. A remarkable characteristic related to low-level resistance to glycopeptides in CoNS is cell wall thickness [27]. Due to its mechanism of action, this characteristic may interfere with daptomycin also, at least in clinical setting or subsequent to extensive use.

\section{Conclusion}

In conclusion, although still relatively infrequent, multiresistant CoNS with reduced susceptibility to glycopeptides are emerging pathogens of clinical concern and should be kept in mind both in empirical and rational therapy of CR-BSI. Surveillance by antibiotyping with attention to multi-resistant profile is mandatory and warning to clinicians is also necessary. Genotyping analysis are useful to discern an epidemic outbreak, which should lead to the implementation of behavioural modifications and, potentially, decontamination may be considered.

\section{Abbreviations}

CLSI: Clinical and Laboratory Standards Institute; CoNS: Coagulase Negative Staphylococci; BSI: Blood stream infection; CR-BSI: Catheter related blood stream infec- tion; EUCAST: European Committee for Antimicrobial Susceptibility testing; f-AFLP: Amplified Fragment Length Polymorphism; ICU: Intensive Care Unit; MIC: Minimum Inhibiotory Concentration; MRSA: Methicillin-resistant Stapylococcus aureus; MDR: Multidrug-resistant.

\section{Competing interests}

The authors declare that they have no competing interests.

\section{Authors' contributions}

$\mathrm{SN}, \mathrm{CF}, \mathrm{MF}, \mathrm{GPT}, \mathrm{AB}$ and FL contributed to the conception, review of the studies and data analysis. $\mathrm{SN}$ and $\mathrm{CF}$ are also involved in drafting the manuscript. $\mathrm{SM} M \mathrm{MCB}, \mathrm{AB}$, $\mathrm{LC}, \mathrm{GB}, \mathrm{MC}$ and $\mathrm{RC}$ contributed in acquisition and interpretation of data. SM and MBC also performed antimicrobial suceptibility tests. MF also performed genetic analysis. All authors approved the final version of the manuscript

\section{Acknowledgements}

We gratefully acknowledge Dr. Alma Viaggi, Dr. Agostino Alessandrina, Dr Maria Cristina Conti and Mario Fede for their contribution to our investigation.

\section{References}

I. Schaberg DR, Culver DH, Gaynes RP: Major trends in the microbial etiology of nosocomial infection. Am J med 1991, 9I(3B):72S-75S.

2. Banerjee SN, Emori TG, Culver DH, Gaynes RP, Jarvis WR, Horan T, Edwards JR, Tolson J, Henderson T, Martone VJ: Secular trends in nosocomial primary bloodstream infections in the United States, 1980-1989. National Nosocomial Infections Surveillance System. Am J Med I 991, 91 (3B):86S-89S.

3. Emory TG, Gaynes RP: An overview of nosocomial infections, including the role of the microbiology laboratory. Clin Microbiol Rev 1993, 6:428-442.

4. Cunningham R: Comparative therapeutic efficacy of teicoplanin and vancomycin in normal and in neutropenic mice infected with Staphylococcus haemolyticus. J antimicrob Chemother 1992, 29:459-66.

5. Menichetti F: Gram-positive infections in neutropenic patients: glycopeptide antibiotics choice. J Antimicrob Chemother 1992, 29:461-3.

6. Nakipoglu Y, Derbentli S, Cagatay AA, Katranci H: Investigation of Staphylococcus strains with heterogeneous resistance to glycopeptides in a Turkish university hospital. BMC Infect Dis 2005, 5(I):3I.

7. Tabe Y, Nakamura A, Igari J: Glycopeptide susceptibility profiles of nosocomial multiresistant Staphylococcus haemolyticus isolates. J Infect Chemother 200I, 7(3):I42-7.

8. Garrett DO, Jochimensen E, Murfitt K, Hill B, McAllister S, Nelson P, Spera RV, Sall RK, Tenover FC, Johnston J, Zimmer B, Jarvis WR: The Emergence of decreased suscepitibility to vancomycin in Staphilococcus Epidermidis. Infect Control Hosp Epidemiol 1999, 20(3): 167-70.

9. Del Bene VE, Jhon JF Jr, Twitty JA, Lewis JW: Antistaphylococcal activity of teicoplanin, vancomycin, and other antimicrobial agents: the significance of methicillin resistance. J Infect Dis 1986, I 54:349-52.

10. Uttley AH, Collins $\mathrm{CH}$, Naidoo J, George RC: Vancomycin-resistant enterococci. Lancet 1988, I (8575-6):57-58.

II. Leclercq R, merlot E, Duval J, Courvalin P: Plasmid-mediated resistance to vancomycin and teicoplanin in Enterococcus faecium. N Engl J Med 1988, 3 19:|57-|6|.

12. Hiramatsu K, Aritaka N, Hanaki H, Kawasaki S, Hosoda Y, Hori S, Fukuchi $Y$, Kobayashi I: Dissemination in Japanese hospitals of 
strains of Staphylococcus aureus heterogeneously resistantt to vancomycin. Lancet 1997, 350:1670-1673.

13. Smith TL, Pearson ML, Wilcox KR, Cruz C, Lancaster MV, RobinsonDunn B, Tenover FC, Zervos MJ, Band JD, White E, Jarvis WR: Emergence of vancomycin resistance in staphylococcus aureus. N Engl I Med 1999, 340(7):493-50I.

14. Webster D, Rennie RP, Brosnikoff CL, Chui L, Brown C: Methicillinresistant Staphylococcus aureus with reduced susceptibility to vancomycin in Canada. Diagn Microbiol Infect Dis 2007, 57(2): $|77-8|$.

15. Howe RA, Wootton M, Walsh TR, Bennett PM, MacGowan AP: Expression and detection of hetero-vancomycin resistance in Staphylococcus aureus. J Antimicrob Chemother 1999, 44(5):675-8.

16. CLSI: Performance Standards for antimicrobial susceptibility testing; Eighteenth Informational Supplement. In CLSI document M100-S I 8 Wayne, PA: Clinical and Laboratory Standards Institute; 2008.

17. Laboratory Detection of Vancomycin-Intermediate/Resistant Staphylococcus aureus (VISA/VRSA) CDC Updated 2006 [http://www.cdc.gov/ncidod/dhqp/ar visavrsa labFAQ.html].

18. Sneath PHA, Sokal RR: Numerical Taxonomy: Principles and Practice of numerical taxonomy. San Francisco, WH Freeman; 1973.

19. Struelens MJ, De Gheldre Y, Deplano A: Comparative and library epidemiological typing systems: outbreak investigations versus surveillance systems. Infect Control Hosp Epidemiol 1998, 19(8):565-9.

20. Srinivasan A, Dick JD, Perl TM: Vancomycin resistance in staphylococci. Clin Microbiol Rev 2002, I 5(3):430-8.

21. Schwalbe RS, Stapleton JT, Gilligan Ph: Emergence of vancomycinresistance in coagulase-negative staphylococci. N Engl J Med 1987, 3 16(15):927-931.

22. Biavasco F, Vignaroli C, Varaldo PE: Glycopeptide Resistance in Coagulase-Negative Staphylococci. Eur J Clin Microbiol Infect Dis 2000, 19:403-4I7.

23. Garret Denise O, Jochimsen Elise, Murfitt Kate, Hill Berta, McAllinster Sigrid, Nelson Pat, Spera Richard V, Small Richard K, Tenover Fred C, Johnston Judy, Zimmer Barbara, Jarvis William R: The Emergence of Decreased Susceptibility to Vancomycin in Staphylococcus Epidermidis. Infection Control and Hospital Epidemiology 1999, 20(3):167-170.

24. Diekema DJ, Pfaller MA, Schmitz FJ, Smayevsky J, Bell J, Jones RN Beach M, SENTRY Partcipants Group: Survey of infections due to Staphylococcus species: frequency of occurrence and antimicrobial susceptibility of isolates collected in the United States, Canada, Latin America, Europe, and the Western Pacific region for the SENTRY Antimicrobial Surveillance Program, 1997-1999. Clin Infect Dis 200I, 32(SuppI 2):SI I4-32.

25. Alamo DA, Cereda FR, Tosin I, Miranda AE, Sader SH: Antimicrobial susceptibility of coagulase-negative Staphylococci and characterization of isolates with reduced susceptibility to glycopeptides. Diagn Microbiol Infect Dis 1999, 34: I85-91.

26. Yoko T, Ayaco N, Jun I: Glycopeptide susceptibility profiles of nosocomial multiresistant Staphylococcus haemolyticus isolates. J Infect Chemoter 200I, 7:142- I47.

27. Ferreira Nunes AP, Teixeira Lúcia Martins, lorio Natália Lopes Pontes, Bastos Carla Callegário Reis, Leila de Sousa Fonseca, SoutoPadrón Thaís, dos Santos Kátia Regina Netto: Heterogeneous resistance to vancomycin in Staphylococcus epidermidis, Staphylococcus haemolyticus and Staphylococcus warneri clinical strains: characterization of glycopeptides susceptibility profiles and cell wall thickening. International Journal of Antimicrobial Agents 2006, 27:307-315.

28. FDA ALERT 3//6/2007 [http://www.fda.gov/medwatch/safety/ 2007/safety07.htm\#Zyvox]

29. Gòmez J, Garcì-Vazquez E Baños R, Canteras M, Ruiz J, Baños V, Herrero JA, Valdés M: Predictors of mortality in patients with methicillin-resistant staphilococcus aureus (MRSA) bacteraemia: the role of empiric antibiotic therapy. Eur J Clin Microbiol Infect Dis 2007, 26:239-245.

30. Grau S, Mateu-de Antonio J, Soto J, Marín-Casino M, Salas E: Pharmacoeconomic evaluation of linezolid versus teicoplanin in bacteremia by Gram-positive microorganisms. Pharm World Sci 2005, 27(6):459-64.
31. Fowler VG, Boucher HW, Corey GR, Abrutyn E, Karchmer AW, Rupp ME, Levine DP, Chambers HF, Tally FP, Vigliani GA, Cabell CH, Link AS, DeMeyer I, Filler SG, Zervos M, Cook P, Parsonnet J, Bernstein JM, Price CS, Forrest GN, Fatkenheuer G, Gareca M, Rehm SJ, Brodt HR, Tice A, Cosgrove SE: Daptomycin versus standard therapy for bacteremia and endocarditis caused by Staphylococcus aureus. N Engl J Med 2006, 335(7):653-665.

\section{Pre-publication history}

The pre-publication history for this paper can be accessed here:

http://www.biomedcentral.com/1471-2334/9/83/prepub
Publish with Biomed Central and every scientist can read your work free of charge

"BioMed Central will be the most significant development for disseminating the results of biomedical research in our lifetime. "

Sir Paul Nurse, Cancer Research UK

Your research papers will be:

- available free of charge to the entire biomedical community

- peer reviewed and published immediately upon acceptance

- cited in PubMed and archived on PubMed Central

- yours - you keep the copyright
BioMedcentral 